\title{
Targeting innate immunity for tuberculosis vaccination
}

\author{
Shabaana A. Khader, ${ }^{1}$ Maziar Divangahi, ${ }^{2}$ Willem Hanekom, ${ }^{3}$ Philip C. Hill, ${ }^{4}$ Markus Maeurer, ${ }^{5,6}$ Karen W. Makar, ${ }^{3}$ \\ Katrin D. Mayer-Barber, ${ }^{7}$ Musa M. Mhlanga, ${ }^{8}$ Elisa Nemes, ${ }^{9}$ Larry S. Schlesinger, ${ }^{10}$ Reinout van Crevel, ${ }^{11}$ \\ Raman (Krishna) Vankayalapati, ${ }^{12}$ Ramnik J. Xavier, ${ }^{13,14,15,16}$ and Mihai G. Netea, ${ }^{11,17}$ on behalf of the Bill and Melinda Gates \\ Foundation Collaboration for TB Vaccine Discovery Innate Immunity Working Group ${ }^{18}$
}

\begin{abstract}
'Department of Molecular Microbiology, Washington University School of Medicine in St. Louis, St. Louis, Missouri, USA. ²Meakins-Christie Laboratories, Department of Medicine, Department of Microbiology and Immunology, and Department of Pathology, McCill International TB Centre, McCill University Health Centre, Montreal, Quebec, Canada. 'Bill \& Melinda Gates Foundation, Seattle, Washington, USA.

${ }^{4}$ Centre for International Health, Department of Preventive and Social Medicine, University of Otago Medical School, Dunedin, New Zealand. ${ }^{5}$ Department of Oncology/Haematology, Krankenhaus Nordwest

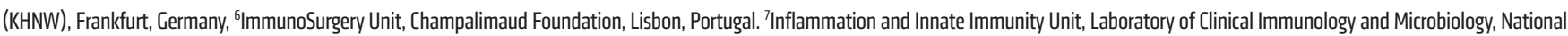
Institute of Allergy and Infectious Diseases, NIH, Bethesda, Maryland, USA. ${ }^{8}$ Division of Chemical Systems \& Synthetic Biology, Institute for Infectious Disease \& Molecular Medicine (IDM), Faculty of Health Sciences, Department of Integrative Biomedical Sciences, and ${ }^{9}$ South African Tuberculosis Vaccine Initiative, Institute of Infectious Disease and Molecular Medicine and Division of Immunology, Department of Pathology, University of Cape Town, Cape Town, South Africa. ${ }^{10}$ Texas Biomedical Research Institute, San Antonio, Texas, USA. "Department of Internal Medicine and Radboud Center for Infectious Diseases, Radboud University Medical Center, Nijmegen, Netherlands. ${ }^{2}$ Department of Pulmonary Immunology, Center for Biomedical Research, University of Texas Health Science Center at Tyler, Tyler, Texas, USA. ${ }^{3}$ Broad Institute of MIT and Harvard, Cambridge, Massachusetts, USA. ${ }^{14}$ Center for Computational and Integrative Biology and ${ }^{15}$ Castrointestinal Unit and Center for the Study of Inflammatory Bowel Disease, Massachusetts General Hospital and Harvard Medical School, Boston, Massachusetts, USA. ${ }^{16}$ Center for Microbiome Informatics and Therapeutics, Massachusetts Institute of Technology, Cambridge, Massachusetts, USA. ${ }^{17}$ Department for Cenomics \& Immunoregulation, Life and Medical Sciences Institute (LIMES), University of Bonn, Bonn, Cermany. ${ }^{18 T h e ~ W o r k i n g ~ G r o u p ~ i s ~}$ detailed in Supplemental Acknowledgments.
\end{abstract}

\begin{abstract}
Vaccine development against tuberculosis (TB) is based on the induction of adaptive immune responses endowed with long-term memory against mycobacterial antigens. Memory B and T cells initiate a rapid and robust immune response upon encounter with Mycobacterium tuberculosis, thus achieving long-lasting protection against infection. Recent studies have shown, however, that innate immune cell populations such as myeloid cells and NK cells also undergo functional adaptation after infection or vaccination, a de facto innate immune memory that is also termed trained immunity. Experimental and epidemiological data have shown that induction of trained immunity contributes to the beneficial heterologous effects of vaccines such as bacille Calmette-Guérin (BCG), the licensed TB vaccine. Moreover, increasing evidence argues that trained immunity also contributes to the anti-TB effects of BCC vaccination. An interaction among immunological signals, metabolic rewiring, and epigenetic reprogramming underlies the molecular mechanisms mediating trained immunity in myeloid cells and their bone marrow progenitors. Future studies are warranted to explore the untapped potential of trained immunity to develop a future generation of TB vaccines that would combine innate and adaptive immune memory induction.
\end{abstract}

\section{Introduction}

Vaccination has made an enormous contribution to improving human and animal health over the last two centuries (1-6). Despite progress in developing vaccines against a large number of infections, vaccine development against global chronic infectious disease threats such as tuberculosis (TB), HIV infection, and malaria has remained a challenge, and achievements to date have been modest.

TB kills more than 1.3 million people a year (7). The current vaccine, bacille Calmette-Guérin (BCG), is an attenuated strain of Mycobacterium bovis and has been in use since the 1920s; approximately 100 million infants receive BCG annually. BCG is efficient in children, with greater than $50 \%$ protection against lung disease and more than $80 \%$ protection against disseminated forms of $\mathrm{TB}(8)$. Importantly, however, children do not spread TB, while adolescents

Conflict of interest: RX is a consultant for Novartis and Nestlé, and cofounder of Inana Therapeutics and Celsius Therapeutics.

Copyright: (c) 2019, American Society for Clinical Investigation.

Reference information: / Clin Invest. 2019;129(9):3482-3491.

https://doi.org/10.1172/JCI128877. and adults do (9). Unfortunately, BCG has shown variable and mostly poor protection against $\mathrm{TB}$ in adolescents and adults. Therefore, new vaccines that target both children and adult populations are needed.

Multiple TB vaccine candidates have entered clinical trials. In 2018, two major clinical advances were reported. The novel subunit vaccine $\mathrm{M} 72 / \mathrm{ASO}_{\mathrm{E}}$ was shown to prevent progression to pulmonary TB disease in adults with prior immunological sensitization to Mycobacterium tuberculosis (Mtb), measured by QuantiFERON-TB (QFT; QIAGEN), with an efficacy of 54\% (95\% CI, $3 \%-78 \%)(10)$. In a second trial, BCG revaccination in adolescence was shown to protect QFT-negative adolescents against sustained QFT conversion, indicative of established $M t b$ infection, with an efficacy of $45 \%$ (95\% CI, 6\%-68\%) (5). These results require confirmation but provide early proof of principle that adolescent/ adult TB vaccination strategies may be feasible. In the preclinical space, a CMV-vectored vaccine has shown the best protection reported to date against $M t b$ challenge in nonhuman primates and is on track to enter clinical trials (11).

Despite this progress, new TB vaccine discovery and development remain impeded by our limited understanding of host re- 


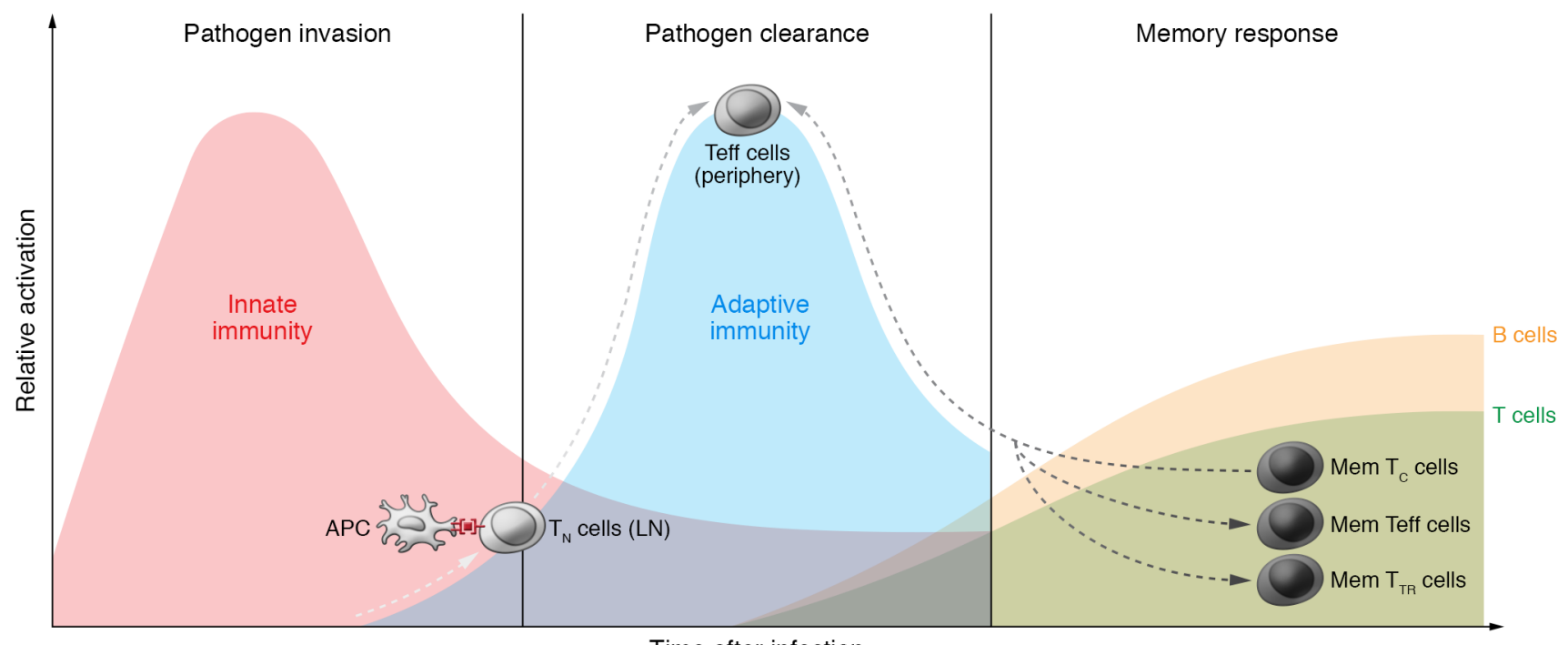

Time after infection

Figure 1. Sequential activation of innate and adaptive immunity during infection, and activation of a long-term memory response through $\mathrm{T}$ and $\mathrm{B}$ lymphocytes. The life cycle of memory T cells is depicted in corresponding phases of pathogen infection, clearance, and memory response. In secondary lymphoid organs (e.g., lymph node [LN]), APCs process and present microbial antigen to naive $T$ ( $T_{N}$ ) cells and convert them to effector $T$ (Teff) cells. These Teff cells then migrate to infected peripheral tissues (e.g., lung) to control infection. After pathogen clearance, Teff cells substantially contract, but a small fraction of antigen-experienced Teff cells convert to: effector memory T (Mem Teff) cells, circulating between lymphoid organs and peripheral tissues; tissue-resident memory $\mathrm{T}\left(\mathrm{Mem} \mathrm{T}_{\mathrm{TR}}\right.$ ) cells, residing in peripheral tissues; and central memory $\mathrm{T}\left(\mathrm{Mem}_{\mathrm{C}}\right.$ ) cells, which are long-lived and reside in secondary lymphoid organs.

sponses required to control $M t b$, as well as technical gaps such as animal models that are predictive of human immune responses (3). Whereas traditional approaches to TB vaccine development have focused on optimal engagement of the adaptive immune system, mainly due to the memory capacity of $\mathrm{T}$ cells, according to an emerging paradigm the innate immune system has memorylike capacity (termed trained immunity) $(2,6)$ and plays a role in the "nonspecific" beneficial effects of vaccines. In this Review, we explore the rationale for targeting the innate immune mechanisms for improving vaccines against TB.

\section{Classical design of vaccines: targeting adaptive immunity}

Classical design of vaccines is based on priming of antigenspecific naive B and T cells to generate memory B and T cells, which are able to initiate a rapid and robust immune response upon encounter with the same pathogen, thus achieving longlasting protection from initial infection or disease (Figure 1).

$\mathrm{B}$ and $\mathrm{T}$ cell specificity is dependent on somatic rearrangement of genes coding for receptors that recognize specific epitopes and can distinguish self and non-self (12). When naive lymphocytes are presented with their cognate antigen in the appropriate cytokine milieu, they become activated and undergo clonal expansion, thereby exponentially increasing the magnitude of antigen-specific cells over several days. During priming, B and T cell transcriptional profiles are epigenetically modulated and drive differentiation into effector and memory cells (13) (Figure 1). Activated B cells undergo immunoglobulin class switching and somatic hypermutation to produce high-affinity Abs with diverse effector functions. Activated T cells rapidly produce effector molecules, such as cytokines and cytotoxic mediators (14), and experience metabolic reprogramming (15). Activation-induced changes in gene expres- sion also affect expression of surface molecules that mediate homing to lymphoid and peripheral tissues, where pathogens are most likely to be encountered. Effector T cells migrate to the infected tissues to control infection, then contract, leaving a smaller pool of long-lived memory cells, mostly residing in the bone marrow (BM) or lymphoid tissues. If they encounter the same pathogen again, antigen-specific memory cells react faster and more efficiently than during primary infection. Less differentiated $\mathrm{T}$ cells, such as memory stem cells (16) and central memory cells (17), retain high proliferative capacity, associated with long-term anamnestic responses after vaccination (18). More differentiated T cells, such as circulating effector (17) and tissue-resident memory T cells (19), exhibit strong effector functions that are important for immediate protection against pathogens (20) (Figure 1). A balanced induction of $\mathrm{T}$ cells endowed with long-term memory as well as the capacity to rapidly migrate to the lung parenchyma is important for $\mathrm{T}$ cellmediated protection against TB (21).

Antigen-presenting cells, amplification of the response through costimulatory molecules, and $\mathrm{T}$ cell receptor signal strength play key roles in shaping $\mathrm{T}$ and $\mathrm{B}$ cell differentiation and functional polarization during priming $(22,23)$. Regardless, $\mathrm{T}$ cells maintain both functional and differentiation plasticity, being able to switch expression of lineage-regulating transcription factors (24) as well as to dedifferentiate (25). Vaccine formulation and delivery shape the distinctive features of adaptive immune responses, and antigen selection determines the specificity and breadth of vaccineinduced immune responses. Highly expressed immunodominant antigens are commonly included in vaccines, but antigen selection has not been straightforward for TB vaccines. $M t b$ contains approximately 4000 genes (26), many of which are expressed differentially during infection (27). T cell epitopes are conserved in $M t b$ (28), suggesting an evolutionary advantage of T cell recog- 
nition, possibly to drive lung tissue damage and TB transmission, but $\mathrm{T}$ cell recognition could also contribute to protection, since most individuals do not develop TB following exposure. Indeed, decoy mechanisms such as export of mycobacterial antigens from infected cells (29) divert T cell recognition to promote bacterial persistence. The development of MTBVAC, a live attenuated vaccine retaining most antigens present in virulent $M t b$, therefore, represents a radical approach to circumvent biased antigen selection (30).

The functional attributes of protective $\mathrm{T}$ cells are not fully understood. Several lines of evidence in humans and animal models point toward a protective role for Th1 and Th17 cells (21). A direct comparison of six candidate TB vaccines in clinical testing showed remarkable similarities in the Th1 functional profiles of vaccineinduced $\mathrm{CD}^{+} \mathrm{T}$ cells, with $\mathrm{M} 72 / \mathrm{ASO}_{\mathrm{E}}$ inducing the highest magnitude of memory responses (31). Whether such potent $\mathrm{T}$ cell responses are associated with the partial protection against TB afforded by M72/ASO1 ${ }_{\mathrm{E}}$ (10) will be established in correlates of protection studies. On the other hand, accumulating evidence indicates that unleashing $\mathrm{T}$ cell responses via reprogramming $\mathrm{T}$ cell metabolism (32) or checkpoint inhibitors (e.g., programmed cell death 1 [PD-1]) $(33,34)$ increases susceptibility to TB.

The role of B cells in mediating TB vaccine efficacy is emerging but incompletely understood. Animal models and human TB provide clear evidence that proliferating antigen-specific B cells localize within protective granulomas, highly specialized spatial structures that develop for the containment of infection (35). More recent evidence suggests that Abs from latently infected individuals drive enhanced phagolysosomal maturation, inflammasome activation, and macrophage killing of intracellular $M t b$ when compared with Abs isolated from TB patients (36). These differences in functional outcomes have been linked to the Fc functional profiles of the Abs, selective binding to the Fc $\gamma$ receptor Fc $\gamma$ RIII, and distinct $A b$ glycosylation patterns found in Abs from healthy persons with $M t b$ infection compared with TB patients. Also, while $M t b$-exposed individuals and patients with TB generate $\mathrm{Ab}$ responses against mycobacterial surface antigens and are protective against cell invasion, the inhibitory activity of anti-Mtb Abs appears to be limited to the IgA isotype (37). Recently, individuals highly exposed to $M t b$ who tested negative by QFT and tuberculin skin test (TST) were considered "resisters" and shown to possess IgM, classswitched IgG Ab responses, and non-IFN- $\gamma \mathrm{T}$ cell responses to $M t b$-specific proteins (38). In TB, localization of CXCR5expressing $\mathrm{CD}^{+} \mathrm{T}$ cells within $\mathrm{B}$ cell follicles in TB granulomas near $M t b$-infected macrophages mediates superior $M t b$ control $(35,39)$. Thus, it is possible that in addition to T cells, B cells also engage and participate in effective generation of vaccineinduced immunity against TB.

\section{Vaccine choice and delivery impact immune responses}

Vaccine formulation (e.g., choice of adjuvants and live attenuated vectors) can affect the magnitude and functional quality of vaccine-induced adaptive immune responses (40); therefore, knowledge about appropriate responses to a pathogen is essential for rational vaccine design. Adjuvants and live vectors provide a danger signal for a large variety of innate immune cells, such as neutrophils, macrophages, monocytes, and DCs. In the traditional setting, adjuvants initiate priming and expansion of naive $\mathrm{T}$ cell responses through DC activation that leads to DC maturation and migration to the draining lymph node, where they prime naive $B$ and $T$ cells. Depending on the pattern recognition receptor (PRR) activated on DCs, different costimulatory and cytokine milieus are induced, thus affecting differentiation and polarization of adaptive immunity $(41,42)$. However, by broadly activating diverse innate immune cell types, adjuvants can also alter the activation state and epigenetic reprogramming of innate immune cells, including those potentially exposed to $M t b$.

In addition to adjuvants, the choice of vaccine delivery (such as route and schedule of immunizations) can influence tissue localization and longevity of vaccine-induced immune responses $(43,44)$. This appears particularly important in TB, where $\mathrm{T}$ cells homing to the lung parenchyma are associated with superior protection compared with those that remain intravascular in a mouse model $(45,46)$. Intravenous or lung mucosal BCG administration is associated with reduced TB pathology compared with intradermal vaccination in nonhuman primates (47, 48). Mucosal vaccination with an attenuated $M t b$ vaccine candidate, $M t b$ sigH, induces B cell-harboring lymphoid follicles in the lung that serve as local immune structures to mediate highly effective $\mathrm{T}$ cell immune responses and better protection than even mucosal BCG vaccination (39). Live BCG can also induce a specific cytokine profile in human APCs to promote $\mathrm{T}$ follicular helper $\left(\mathrm{T}_{\mathrm{FH}}\right)$ cell differentiation (49).

Another TB vaccine candidate based on a recombinant CMV vector (RhCMV/TB) conferred complete protection in approximately $40 \%$ of nonhuman primates (11). Although this vaccine, administered subcutaneously, induced and maintained highly differentiated circulating and tissue-resident memory $\mathrm{T}$ cells, protection was associated with innate cell (particularly neutrophil) activation (11). Furthermore, as discussed further below, targeting innate immune activation pathways, specifically CD4O/ CD4OL and $\mathrm{CD} 103^{+}$DCs in mouse models, can induce rapid antigen-specific $\mathrm{T}$ cell responses, formation of $\mathrm{B}$ cell lymphoid follicles, and superior near-sterilizing immunity in the lungs of BCG-vaccinated mice (50). These studies together provide new avenues to target innate immune responses to improve $\mathrm{T}$ and $\mathrm{B}$ cell responses for TB vaccines. Ultimately, adaptive and innate immune responses work in a coordinated fashion during both priming and effector phases of a successful immune response.

\section{Available adjuvants and their functions}

Innate immunity plays a key role in $\mathrm{TB}$ vaccine responses through an increase in the function of antigen-presenting cells by vaccine adjuvants. Although some types of vaccines, such as live attenuated (e.g., yellow fever), whole cell (e.g., BCG), and certain killed vaccines (e.g., inactivated polio vaccine [IPV]), do not need specialized adjuvants as part of the vaccine formulation, most if not all subunit/protein vaccines require adjuvants to trigger innate immune activation for efficient humoral and/or $\mathrm{T}$ cell responses for effective vaccine efficacy (51). Few adjuvants are licensed for use in humans, including the aluminum-containing adjuvants CPG 1018, MF59, and AS01, but recently, there has been progress in advancing more formulations for clinical use. 
Adjuvants are categorized based on composition, delivery systems, and their ability to trigger innate immune activation (52). Aluminum-containing adjuvants (e.g., alum) have been used for more than 70 years and are suggested to stimulate the inflammasome (53), but their mode of action is still not fully characterized. Oil-in-water emulsions such as MF59 and ASO3 attract neutrophils and traffic antigen to lymph nodes (54). TLR4 agonists mimic specific danger signals (e.g., bacterial LPS, microbial DNA, or single- or double-stranded RNA) that trigger surface or intracellular receptors to induce innate pathways in antigen-presenting cells to program adaptive immunity. The plant-derived saponin QS21 elicits the release of alarmins, which activate DCs and the inflammasome to generate $\mathrm{CD}^{+} \mathrm{T}$ cell responses and IgG1 and IgG3 Ab production $(51,55)$. Thus, one key commonality of many adjuvants is the stimulation of IL-1 cytokine family members (56). The mechanisms associated with the performance of licensed adjuvanted vaccines is the subject of a recent review (57).

There are several experimental adjuvanted subunit TB vaccines in clinical testing (see Global Clinical Portfolio of TB Vaccine Candidates, http://www.aeras.org/pages/global-portfolio). The M72/AS01 vaccine formulation, which was recently reported to have efficacy against pulmonary $\mathrm{TB}$, contains the combination adjuvant AS01, which is part of the successful commercial vaccine Shingrix (GSK) and a recombinant fusion protein antigen (10). ASO1 is composed of QS21, liposomes, and the TLR4 agonist monophosphoryl lipid A (MPL; a detoxified derivative of bacterial lipopolysaccharide). The proposed mechanism of action of the adjuvant involves triggering naturally occurring innate immune pathways including early release of alarmins by innate immune cells and IFN- $\gamma$ by NK cells $(58,59)$.

The adjuvant glucopyranosyl lipid adjuvant (GLA) is a synthetic lipid A molecule and TLR4 agonist formulated into a stable oil-in-water nanoemulsion (SE) that drives Th1 immune responses (55). GLA-SE is part of an experimental TB vaccine formulation with fusion of four Mtb protein antigens called ID93 (60). Firstin-human studies of ID93 showed improved $\mathrm{Ab}$ and $\mathrm{CD}^{+} \mathrm{T}$ cell responses in GLA-SE-adjuvanted versus unadjuvanted vaccine (61). The vaccine was safe and immunogenic in previously BCG-vaccinated adults (62). A phase IIa clinical trial in treated TB patients (NCT02465216) has completed, and a lyophilized formulation has entered phase I (NCT03722472).

In addition, two cationic particulate adjuvants, IC31 (Valneva Technologies) and CAF01 (Statens Serum Institut), are being tested among experimental TB vaccines. A vaccine comprising the H56 fusion protein formulated with IC31 has completed phase I testing $(63,64)$ and is being evaluated in a phase II clinical trial for preventing TB recurrence (NCT03512249). A different IC31adjvuanted subunit vaccine, H4:IC31, was evaluated for prevention of $M t b$ infection (5). Adjuvant formulation is also important, as shown for the novel TB vaccine candidate $\mathrm{M72} / \mathrm{ASO1}_{\mathrm{E}}$. Adjuvants ASO1 and ASO2 contain the same components (MPL and QS21) formulated in either liposomes (AS01) or oil-in-water emulsion (ASO2). While both formulations generated potent and durable Ab responses, ASO1 induced a higher magnitude of antigen-specific Th1 responses (65). Thus, the choice of adjuvant for a vaccine candidate should depend on properties tailored to the specific vaccine Target Product Profile (TPP).

\section{Interpretation of trained immunity as innate immune memory}

In addition to the classical role of innate immunity in amplifying T/B cell immune memory through adjuvant activity, recent studies provide evidence that a prolonged increase in the antimicrobial function of innate immune cells can itself contribute to protection from reinfection. This functional reprogramming of innate immune cells such as myeloid and NK cells, termed trained immunity, represents a de facto innate immune memory (6). Trained monocytes and macrophages display functional and epigenetic reprogramming, leading to increased production of cytokines and chemokines, and improved phagocytosis and killing capacity (66). Studies have demonstrated that immunological signals, metabolic rewiring of cell metabolism, and epigenetic reprogramming are integrated, representing the molecular substrates for induction of trained immunity (6). The first step involves immunological signals induced through PRRs such as dectin-1 (in the case of $\beta$-glucans) and NOD2 (for BCG). The molecular link between these signals and the epigenetic changes in the nucleus has recently been attributed to changes in cellular metabolism. An initial study reported that induction of trained immunity is accompanied by a shift from oxidative phosphorylation to glycolysis, or the Warburg effect (67). Subsequent studies have shown that the Krebs cycle is replenished through glutaminolysis, leading to accumulation of succinate and especially fumarate. In turn, fumarate inhibits the KDM5 family of demethylases, which are specific for $\mathrm{H} 3 \mathrm{~K} 4 \mathrm{me} 3$; this inhibition permits the retention of this active histone mark (68). Induction of trained immunity leads to an increase in the mevalonate pathway; mevalonate in turn amplifies this process through an insulin-like growth factor 1 (IGF-1R)/Akt/ mTOR pathway (69) (Figure 2).

The changes described above have an important impact on the epigenetic program of trained myeloid cells. The beststudied epigenetic changes to immune stimuli involve the posttranslational modification of histone tails at promoter and enhancer regions $(70,71)$. In response to training signals, histone acetylation and methylation play an important role in regulation of gene expression and remodeling of the epigenome, which are important molecular mechanisms implicated in modulating innate immune cell signaling (6). Prior to gene expression, the promoters of innate immune genes are "preloaded" with poised RNA polymerase II, and neighboring histones possess active histone marks such as $\mathrm{H} 3 \mathrm{~K} 4 \mathrm{me} 3(72,73)$. A rise in the level of this active mark, as well as other active marks such as H3K4me1 and H3K27Ac, is a hallmark of trained immunity (74). Indeed, training with $\beta$-glucan leads to $\mathrm{H} 3 \mathrm{~K} 4 \mathrm{me} 3$ accumulation at specific locations in the genome (74). H3K4me 3 is directed to specific promoters in the genome by the presence of a class of long noncoding RNAs (lncRNAs) called immune gene-proximal lncRNAs (IPLs) (75). IPLs are positioned within topological associating domains (TADs), regions of enriched chromatin long-range looping interactions bringing together multigene complexes (76). Within TADs, IPLs recruit a histone modification complex, the COMPASS complex, which in turn directs the trimethylation of $\mathrm{H} 3 \mathrm{~K} 4 \mathrm{me} 3(75,77,78)$. IPLs are transcribed in an nuclear factor of activated T cells-dependent (NFAT-dependent) manner. Silencing of IPLs, disruption of the COMPASS complex, or abrogation 


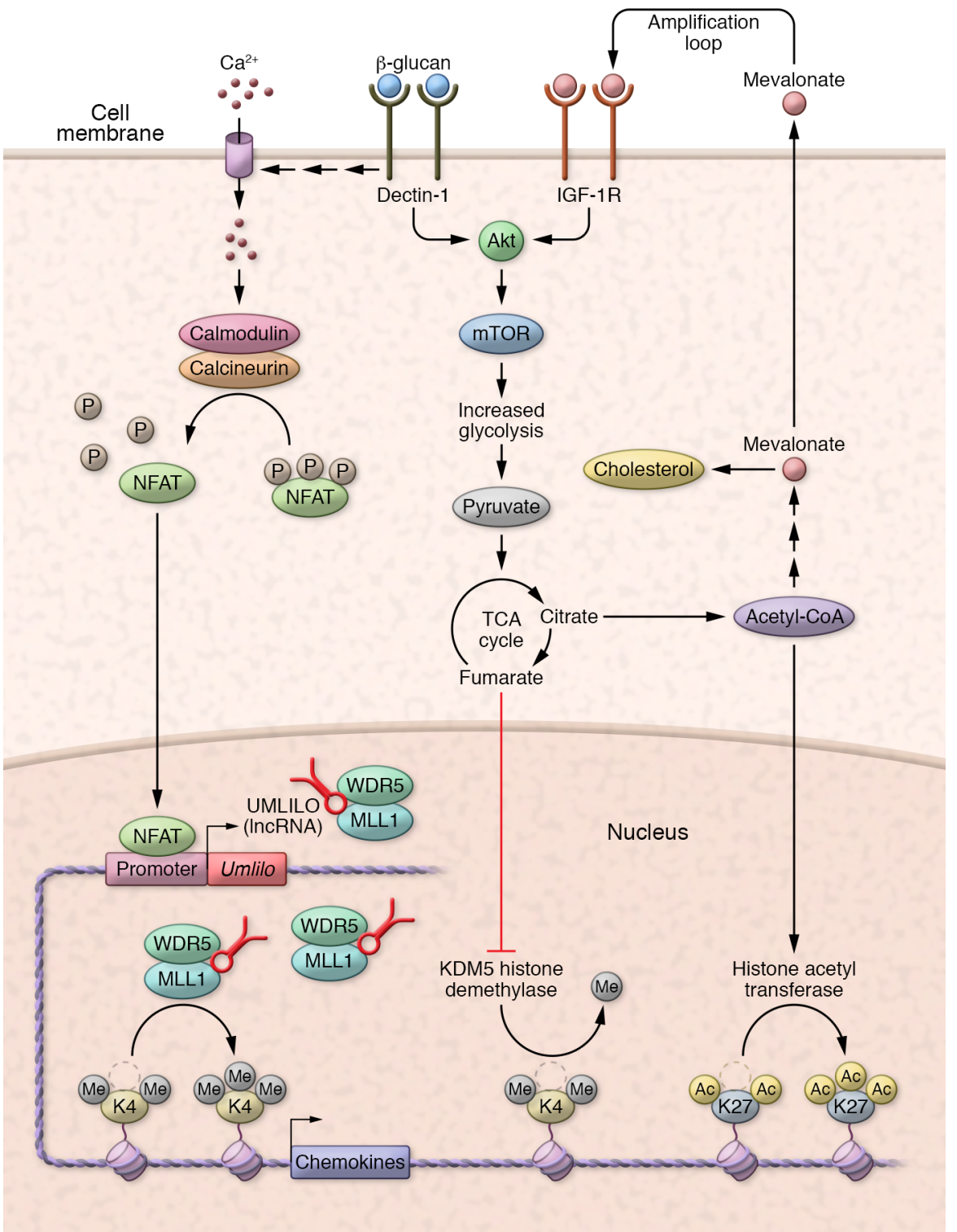

Figure 2. Molecular mechanisms contributing to the induction of trained immunity in myeloid cells. Activation of myeloid cells by microbial $\beta$-glucan or BCG activates PRRs that in turn activate gene transcription, but also cellular metabolism through an Akt/mTOR-dependent pathway. Activation of IncRNAs such as UMLILO determines chromatin changes by activation and transport of histone methyltransferases. Long-term metabolic changes such as fumarate accumulation maintain these changes by inhibiting KDM5 histone demethylases. In turn, mevalonate release amplifies these changes through an IGF-1R-dependent loop. TCA, tricarboxylic acid.

of NFAT signaling results in loss of H3K4me3 accumulation at trained immune genes (75). IPLs are generally conserved across mammals at the sequence and syntenic level; however, a key IPL regulating trained immunity of the chemokine locus is absent in rodents, so CXCL1-3 cannot be trained in mice. Training in mice can be restored by inserting the human IPL (UMLILO) proximal to the mouse chemokines via gene editing (75).

\section{Trained immunity: the impact on myeloid cell progenitors}

Although trained immunity was first established in cells of the mononuclearphagocytelineage (i.e.,monocytes and macrophages), these cells have a relatively short lifespan and are less likely to transmit their memory phenotype to their progeny and provide sustainable protection. In contrast, hematopoietic stem cells (HSCs) are long-lived, with self-renewal properties that reside in the $\mathrm{BM}$, and their transcriptional and functional reprogramming can explain trained immunity induction (Figure 3). In vertebrates, HSCs are generated from endothelial cells in the embryo, which depends on type II IFN signaling (79). The BM is the seat of hematopoiesis, where HSCs constantly undergo asymmetric division, giving rise to the full repertoire of myeloid and lymphoid cell types while maintaining the HSC niche. Importantly, HSCs can directly respond to acute and chronic infections. For example, in a model of acute Escherichia coli infection (80) or chronic Mycobacterium avium infection (81), there was a significant expansion in HSC populations. Similarly, trained immunity induced by BCG vaccination (82) or $\beta$-glucan (83) was recently shown to be mediated through increased myelopoiesis in the BM. Although the exact mechanisms of precursor proliferation/differentiation are not well understood, persistent activation of HSCs can result in BM exhaustion and even complete depletion of HSCs over time, leading to devastating effects on the systemic immune compartment (8486). Thus, the balance between HSC selfrenewal and differentiation must be tightly regulated to maintain the numbers of HSCs for the generation of trained immunity.

Recently, several mechanisms have been proposed to explain HSC activation (87) in the setting of infectious disease, including the following: (i) Direct infection: HSCs are thought to lack the molecular machinery required for phagocytosis. Pathogens including Salmonella, Listeria, Yersinia, M. avium, and BCG are unable to infect HSCs $(81,82,88)$, but $M t b$ infection can gain access to HSCs $(89,90)$. Recent data indicate that $M t b$ infects BM mesenchymal stem cells, which are phagocytic, and bacteria can survive within these cells $(91,92)$. (ii) PRR signaling: HSCs express both cell-surface TLRs (e.g., TLR4, which recognizes bacterial LPS) and cytosolic NOD-like receptors (e.g., NOD2, which recognizes bacterial MDP) that not only play important roles in anti-Mtb immunity, but also drive infection-induced myelopoiesis (generation of monocytes and macrophages), cell mobilization from BM into infection site, and trained immunity (93-96). (iii) Cytokine signaling: Quantitative changes in HSC activity are regulated by cytokines relevant to TB. In vertebrates, both type I IFNs (IFN- $\alpha$ and $-\beta$ ) 


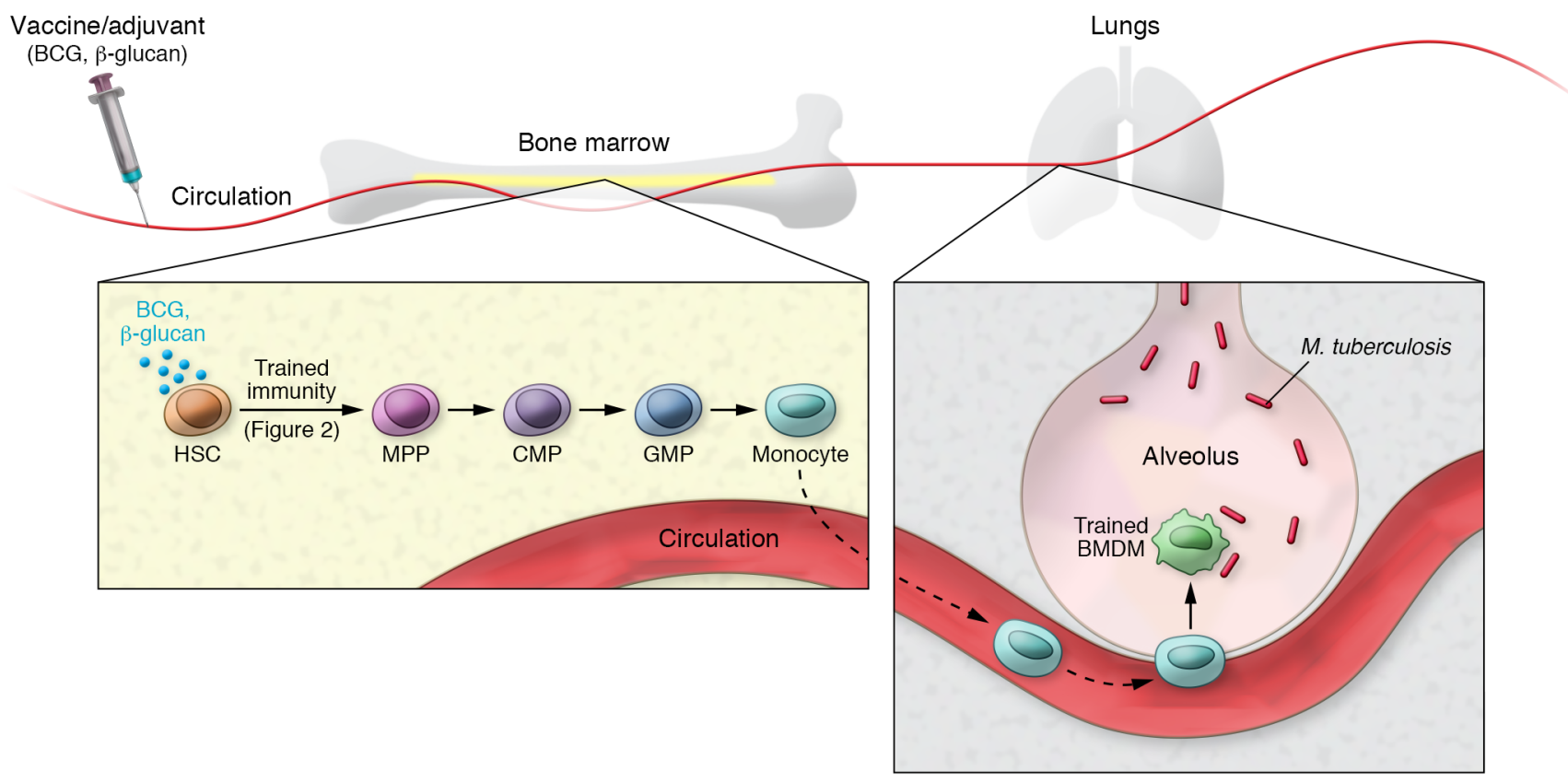

Figure 3. Induction of trained immunity in myeloid cells. Induction of trained immunity by vaccination leads to cellular reprogramming toward a myeloid bias in the BM. Monocytes with rewired transcriptional and epigenetic programs differentiate into trained recruited lung macrophages with increase antimicrobial activity. CMP, common myeloid progenitor; GMP, granulocyte-monocyte progenitor; BMDM, BM-derived macrophage.

and type II IFN (IFN- $\gamma$ ) can increase HSC proliferation (81, 84, $86,97)$, but they have opposing effects in the killing of $M t b$ by macrophages $(98,99)$. Furthermore, increased activation of HSCs following M. avium infection or the generation of HSCmediated trained immunity via BCG intravenous vaccination was shown to be IFN- $\gamma$ dependent, highlighting the pivotal role of IFNs in HSC responses to mycobacterial infection $(81,82)$. While basal levels of IFN signaling are required to maintain the balance between HSC self-renewal and differentiation during homeostasis, chronic exposure of HSCs to IFNs during chronic infections may lead to BM aplasia (79). Interestingly, the role of IFN-dependent pathways in regulating HSC responses and downstream consequences following infection with virulent strains of $M t b$ is still unknown. Although this study indicates that type II IFN is required for generating trained immunity by HSCs after BCG vaccination (82), another study showed that $\beta$-glucaninduced trained immunity by HSCs was mediated via GM-CSF and IL-1 signaling (83). These observations suggest that different stimuli (e.g., live pathogen versus pathogen-derived products) may imprint distinct molecular signatures in HSCs that each lead to trained immunity.

The molecular mechanisms required for inducing trained immunity in the BM require the same epigenetic rewiring events described earlier for mature myeloid cells. It was recently demonstrated that BCG can reprogram HSCs toward myelopoiesis, leading to generation of protective monocytes/macrophages against subsequent $M t b$ infection. Most importantly, this protective signature was transmitted from HSCs to multipotent progenitors (MPPs) to monocytes and macrophages. Epigenetic analysis of BCG-trained, BM-derived macrophages indicates that the H3K4me3 and H3K27Ac marks are significantly enriched for genes associated with resistance to $M t b$ infection. These results provide a mechanism for how in vivo BCG vaccination epigenetically primes $\mathrm{BM}$-derived macrophages to initiate a robust protective response against $M t b$ infection (82). These data are supported by studies describing similar mechanisms at the HSC level induced by $\beta$-glucan (83).

\section{Targeting NK memory for TB vaccines}

NK cells are prominent components of the innate immune system that play a central role in resistance to microbial pathogens. NK cells protect against viruses, bacteria, and parasites by destroying infected cells and secreting cytokines that shape the adaptive immune response (100-103). NK cells contribute to immunity against $M t b$ infection by lysing $M t b$-infected human monocytes and alveolar macrophages and upregulating $\mathrm{CD} 8^{+} \mathrm{T}$ cell responses (104, 105). The pleural fluid of TB patients is enriched for NK cells, which are the predominant source of IFN- $\gamma$ (106), and a subpopulation expresses the memory-associated marker CD45RO, thus exerting robust immune responses when stimulated by IL-12 (107). NK cells lyse $M t b$-expanded Tregs (108), and eliminating NK cells at the time of BCG vaccination enhances Treg expansion and inhibits BCG-induced protection against challenge with $M t b$ (109). Accordingly, in mice, memory-like NK cells develop during BCG vaccination, expand, and provide protection against challenge with $M t b$ (110). Recently, a multicohort study found higher numbers of cytotoxic NK cells in persons with latent TB than in uninfected individuals. These NK cells decreased during the progression from latent infection to active disease and returned to the baseline level after TB treatment, suggesting a protective role for NK cells during $M t b$ infection (111). BCG revaccination of adults with latent TB infection also induces long-lived BCG-reactive NK cell responses (112). Studies suggest that NK cells distinguish antigens, and memory NK cells expand and protect against viral pathogens (113-115). These stud- 
ies provide new evidence that memory-like NK cells survive longterm and could be targeted to promote vaccine-induced protective immunity against $M t b$ infection. A comprehensive evaluation of the role of memory-like NK cell phenotype and function in household contacts who develop active TB will improve our understanding of the protective mechanisms against infection and subsequently facilitate development of interventions to prevent development of active TB. Further understanding of these mechanisms will also lay the groundwork for developing novel methods to stimulate memorylike NK cell-mediated immunity against TB.

\section{Insights gained from BCG vaccination in human studies}

Historically, it was not known whether the benefits of BCG were conferred predominantly through prevention of $M t b$ infection or through prevention of progression to TB disease in those infected. Autopsy data from 150 people and national tuberculin data supported the conclusion that there was no evidence to support the suggestion that BCG vaccination can prevent the establishment of infection in an exposed human (116). Since there was no difference in TST positivity between BCG-vaccinated and unvaccinated individuals, but BCG vaccination was accompanied by differences in TB pathology, it was assumed that BCG protected mainly against progression to disease.

With the introduction of IFN- $\gamma$ release assays (IGRAs), which assess the $\mathrm{T}$ cell response to $M t b$ antigens that are not secreted by BCG, protection against $M t b$ infection could be assessed without confounding test results based on BCG itself. Soysal et al. studied more than 900 child household TB case contacts in Turkey and found a significant reduction in ELISPOT positivity in those who were BCG vaccinated (117). A subsequent systematic review (118) by Roy et al. confirmed this result across 14 cross-sectional studies, with an estimated protection against infection of $19 \%$ (95\% CI, 8\%-29\%). Longitudinal studies whereby an IGRA test was repeated after about 3 months in those initially negative, suggested that the protective effect is even greater in relation to a specific exposure. Hill et al. showed that BCG vaccination was associated with a 50\% (95\% CI, 20\%-100\%) reduction in the risk of ELISPOT conversion after 3 months in Gambian contacts (119). A remarkably similar estimate (45\%; 95\% CI, 24\%-60\%) was obtained by Verrall et al. in Indonesian case contacts (120). Furthermore, in the Indonesian contacts, BCG protection decreased with increasing exposure, suggesting that the immune mechanism for protection could be overcome with increased exposure to the pathogen. This latter finding could have implications for understanding different levels of protection from BCG across populations with varying intensity of $M t b$ transmission, protection being weakest in high-TB-burden settings (121). In addition, the study by Verrall et al. found evidence of BCG protection against infection up until the age of 30 years, which is consistent with recent studies suggesting that BCG protection against TB disease lasts at least 20 years when given at birth or at school age $(122,123)$.

A recent randomized controlled trial among adolescents in South Africa provided further support for a protective effect of BCG against Mtb infection. Among 990 HIV- and QFTnegative adolescents, sustained QFT conversion was $11.2 \%$ in those who received placebo and $6.7 \%$ among those who were
BCG revaccinated over 2 years of follow-up, representing a $45.5 \%$ reduction $(5)$.

The mechanisms of protection against $M t b$ infection induced by BCG vaccination are likely to involve innate immune mechanisms, including trained immunity. Interestingly, in the recently published randomized trial that showed a protective effect of BCG revaccination against $M t b$ infection, unrelated respiratory tract infections were also significantly less common $(5,124)$, supporting a strong increase in heterologous protection against infections, which is suggestive of trained immunity. Epidemiological reports suggest protection against nonspecific infections by BCG for up to $4-5$ years after vaccination in infants (125), and experimental studies in humans have shown the persistence of trained immunity for at least one year after BCG vaccination (126). In addition, BCG-induced protection against $M t b$ infection was associated with higher production of proinflammatory cytokines following heterologous stimulation with Streptococcus pneumoniae and E. coli (120), an observation reminiscent of trained immunity $(127,128)$. Thus, while murine studies suggest that training of HSCs requires access to the BM (82), whether intradermal BCG vaccination in humans similarly accesses the $\mathrm{BM}$ is not known and will no doubt be the target of future research. We envision that there are two major pathways that can lead to trained immunity in HSCs during vaccination: (i) a direct pathway, functioning via access of the vaccine (e.g., BCG) in the BM, likely by generation of an inflammatory milieu that leads to HSC training; and (ii) an indirect pathway, via systemic release of exogenous (e.g., PAMPs) or endogenous mediators (e.g., DAMPs, cytokines, colony-stimulating factors). HSCs express receptors that are important in the activation/mobilization of HSCs $(95,129)$. Thus, systemic release of DAMPs as well as cytokines (e.g., GM-CSF, IFN- $\gamma$ ) (81) during vaccination can reach the BM to train HSCs. Following intradermal BCG vaccination in humans, the systemic release of PAMPs, DAMPS, and/or cytokines is most likely responsible for training HSCs.

\section{Harnessing innate immunity for better vaccines}

The studies reviewed above provide evidence that innate immune processes and, in particular, adjuvant activity and trained immunity, are important components of the protective effects of BCG vaccination against TB. A detailed understanding of these processes brings hope that they can be used for the design of new and more effective vaccines.

Which processes may be enlisted for this end? First, understanding the molecular mechanisms that mediate the biological action of adjuvants is a step toward precise fine-tuning of the type of $\mathrm{T}$ or B cell needed in an infection in general, and in TB in particular. For example, understanding the PRR and signaling pathways leading to the induction of a Th1/Th2/Th17/ $\mathrm{T}_{\mathrm{FH}}$ response will permit the design of adjuvants with specific activity toward inducing a particular type of Th response. Second, integration of trained innate immunity within the biological effects of future vaccines holds promise for improved vaccine effectiveness. Indeed, a vaccine that combines improved antimicrobial effects of both innate (trained immunity) and adaptive (classical immune memory) mechanisms would likely be more effective than current vaccines. Third, these developments should improve the outcome 
of vaccination in populations at higher risk from infection, such as the elderly. Indeed, adaptive immune responses are defective at extreme ages, and incorporation of trained immunity into vaccines designed for use in the elderly may circumvent this problem.

In conclusion, future studies are needed to investigate the impact of the induction of trained immunity on vaccination effects. These studies must focus on the mechanisms involved and how to effectively trigger them. We predict that the impact of combining trained immunity with classical adaptive immune memory will be fully realized in the vaccine community in the years to come. Further definition of trained immunity should enhance approaches to our vaccine armamentarium.

\section{Acknowledgments}

The authors thank Nicole Howard (SAK laboratory) and Stephanie Fanucchi (M. Mhlanga laboratory) for formatting the figures and the manuscript. MGN was supported by a European Research Council Advanced Grant (no. 833247) and a Spinoza grant from the Netherlands Organization for Scientific Research. $\mathrm{EN}$ is an International Society for Advancement of Cytometry (ISAC) Marylou Ingram Scholar. This work was supported by Washington University in St. Louis, NIH grants HL105427, AI111914, AI123780, and AI134236, to SAK. MD was supported by the Canadian Institute of Health Research (CIHR) Foundation Grant (FDN-143273) and holds a Fonds de Recherche du Québec-Santé (FRQS) and the Strauss Chair in Respiratory Diseases. RJX was supported by Broad Institute TB program and NIH AI 109725. KDM-B was supported by intramural research program of NIAID. See Supplemental Acknowledgments (supplemental material available online with this article; https://doi. org/10.1172/JCI128877DS1) for details on the Bill and Melinda Gates Foundation Collaboration for TB Vaccine Discovery Innate Immunity Working Group.

Address correspondence to: Shabaana A. Khader, Department of Molecular Microbiology, Washington University in St.Louis, Campus Box 8230, 660 South Euclid Avenue, St.Louis Missouri 631101093, USA. Phone: 314.286.1590; Email: khader@wustl.edu. Or to: Mihai G. Netea, Department of Internal Medicine, Radboud University, Medical Center, PO Box 9101, 6500HB, Nijmegen, Netherlands. Phone: 31.0.24.361.46.52; Email:mihai.netea@ radboudumc.nl.
1. Greenwood B. The contribution of vaccination to global health: past, present and future. Philos Trans R Soc Lond, B, Biol Sci. 2014;369(1645):20130433.

2. Blok BA, Arts RJ, van Crevel R, Benn CS, Netea MG. Trained innate immunity as underlying mechanism for the long-term, nonspecific effects of vaccines. J Leukoc Biol. 2015;98(3):347-356.

3. Ernst JD. Mechanisms of M. Tuberculosis immune evasion as challenges to TB vaccine design. Cell Host Microbe. 2018;24(1):34-42.

4. Izzo AA. Tuberculosis vaccines - perspectives from the NIH/NIAID Mycobacteria vaccine testing program. Curr Opin Immunol. 2017;47:78-84.

5. Nemes E, et al. Prevention of M. Tuberculosis infection with H4:IC31 vaccine or BCG revaccination. N Engl J Med. 2018;379(2):138-149.

6. Netea MG, et al. Trained immunity: a program of innate immune memory in health and disease. Science. 2016;352(6284):aaf1098.

7. World Health Organization. Global Tuberculosis Report. https://www.who.int/tb/publications/ global_report/en/. Accessed August 6, 2019.

8. Mangtani P, et al. Protection by BCG vaccine against tuberculosis: a systematic review of randomized controlled trials. Clin Infect Dis. 2014;58(4):470-480.

9. Swaminathan S, Rekha B. Pediatric tuberculosis: global overview and challenges. Clin Infect Dis. 2010;50 Suppl 3:S184-S194.

10. Van Der Meeren O, et al. Phase $2 \mathrm{~b}$ controlled trial of M72/ASO1 $1_{\mathrm{E}}$ vaccine to prevent tuberculosis. NEngl JMed. 2018;379(17):1621-1634.

11. Hansen SG, et al. Prevention of tuberculosis in rhesus macaques by a cytomegalovirus-based vaccine. Nat Med. 2018;24(2):130-143.

12. Jenkins MK, Chu HH, McLachlan JB, Moon JJ. On the composition of the preimmune repertoire of T cells specific for peptide-major histocompatibility complex ligands. Annu Rev Immunol. 2010;28:275-294.
13. Schmidl C, Delacher M, Huehn J, Feuerer M. Epigenetic mechanisms regulating T-cell responses. J Allergy Clin Immunol. 2018;142(3):728-743.

14. Akondy RS, et al. Origin and differentiation of human memory CD8 T cells after vaccination. Nature. 2017;552(7685):362-367.

15. Almeida L, Lochner M, Berod L, Sparwasser T. Metabolic pathways in T cell activation and lineage differentiation. Semin Immunol. 2016;28(5):514-524.

16. Gattinoni L, et al. A human memory T cell subset with stem cell-like properties. Nat Med. 2011;17(10):1290-1297.

17. Sallusto F, Lenig D, Forster R, Lipp M, Lanzavecchia A. Two subsets of memory T lymphocytes with distinct homing potentials and effector functions. Nature. 1999;401(6754):708-712.

18. Fuertes Marraco SA, et al. Long-lasting stem cell-like memory CD8+ T cells with a naïve-like profile upon yellow fever vaccination. Sci Transl Med. 2015;7(282):282ra48.

19. Sathaliyawala T, et al. Distribution and compartmentalization of human circulating and tissue-resident memory T cell subsets. Immunity. 2013;38(1):187-197.

20. Masopust D, Picker LJ. Hidden memories: frontline memory T cells and early pathogen interception. J Immunol. 2012;188(12):5811-5817.

21. Andersen P, Scriba TJ. Moving tuberculosis vaccines from theory to practice [published online ahead of print May 21, 2019]. Nat Rev Immunol. https://doi. org/10.1038/s41577-019-0174-z.

22. Palucka K, Banchereau J, Mellman I. Designing vaccines based on biology of human dendritic cell subsets. Immunity. 2010;33(4):464-478.

23. Buchholz VR, Schumacher TN, Busch DH. T cell fate at the single-cell level. Annu Rev Immunol. 2016;34:65-92.

24. O'Shea JJ, Paul WE. Mechanisms underlying lineage commitment and plasticity of helper CD4+ T cells. Science. 2010;327(5969):1098-1102.
25. Youngblood B, et al. Effector CD8 T cells dedifferentiate into long-lived memory cells. Nature. 2017;552(7685):404-409.

26. Cole ST, et al. Deciphering the biology of Mycobacterium tuberculosis from the complete genome sequence. Nature. 1998;393(6685):537-544.

27. Coppola M, Ottenhoff TH. Genome wide approaches discover novel Mycobacterium tuberculosis antigens as correlates of infection, disease, immunity and targets for vaccination. Semin Immunol. 2018;39:88-101.

28. Comas I, et al. Human T cell epitopes of Mycobacterium tuberculosis are evolutionarily hyperconserved. Nat Genet. 2010;42(6):498-503.

29. Srivastava S, Grace PS, Ernst JD. Antigen export reduces antigen presentation and limits $\mathrm{T}$ cell control of M. tuberculosis. Cell Host Microbe. 2016;19(1):44-54.

30. Gonzalo-Asensio J, Marinova D, Martin C, Aguilo N. MTBVAC: attenuating the human pathogen of tuberculosis (TB) toward a promising vaccine against the TB epidemic. Front Immunol. 2017;8:1803.

31. Rodo MJ, et al. A comparison of antigenspecific $\mathrm{T}$ cell responses induced by six novel tuberculosis vaccine candidates. PLoS Pathog. 2019;15(3):e1007643.

32. Tzelepis F, et al. Mitochondrial cyclophilin D regulates $\mathrm{T}$ cell metabolic responses and disease tolerance to tuberculosis. Sci Immunol. 2018;3(23):eaar4135.

33. Barber DL, Mayer-Barber KD, Feng CG, Sharpe $\mathrm{AH}$, Sher A. CD4 T cells promote rather than control tuberculosis in the absence of PD-1-mediated inhibition. JImmunol. 2011;186(3):1598-1607.

34. Barber DL, et al. Tuberculosis following PD-1 blockade for cancer immunotherapy. Sci Transl Med.2019;11(475):eaat2702.

35. Slight SR, et al. CXCR5 ${ }^{+} \mathrm{T}$ helper cells mediate protective immunity against tuberculosis. J Clin Invest. 2013;123(2):712-726. 
36. Lu LL, et al. A functional role for antibodies in tuberculosis. Cell. 2016;167(2):433-443.e14.

37. Zimmermann N, et al. Human isotypedependent inhibitory antibody responses against Mycobacterium tuberculosis. EMBO Mol Med. 2016;8(11):1325-1339.

38. Lu LL, et al. IFN- $\gamma$-independent immune markers of Mycobacterium tuberculosis exposure. Nat Med.2019;25(6):977-987.

39. Kaushal D, et al. Mucosal vaccination with attenuated Mycobacterium tuberculosis induces strong central memory responses and protects against tuberculosis. Nat Commun. 2015;6:8533.

40. Seder RA, Darrah PA, Roederer M. T-cell quality in memory and protection: implications for vaccine design. Nat Rev Immunol. 2008;8(4):247-258.

41. Awate S, Babiuk LA, Mutwiri G. Mechanisms of action of adjuvants. Front Immunol. 2013;4:114

42. Knudsen NP, et al. Different human vaccine adjuvants promote distinct antigen-independent immunological signatures tailored to different pathogens. Sci Rep. 2016;6:19570.

43. Draper SJ, Heeney JL. Viruses as vaccine vectors for infectious diseases and cancer. Nat Rev Microbiol. 2010;8(1):62-73.

44. Butler NS, Nolz JC, Harty JT. Immunologic considerations for generating memory CD8 T cells through vaccination. Cell Microbiol. 2011;13(7):925-933.

45. Sakai S, et al. Cutting edge: control of Mycobacterium tuberculosis infection by a subset of lung parenchyma-homing CD4 T cells. J Immunol. 2014;192(7):2965-2969.

46. Sakai S, et al. CD4 T cell-derived IFN- $\gamma$ plays a minimal role in control of pulmonary Mycobacterium tuberculosis infection and must be actively repressed by PD-1 to prevent lethal disease. PLoS Pathog. 5667;2016(5):e1005667.

47. Dijkman K, et al. Prevention of tuberculosis infection and disease by local BCG in repeatedly exposed rhesus macaques. Nat Med. 2019;25(2):255-262.

48. Sharpe S, et al. Alternative BCG delivery strategies improve protection against Mycobacterium tuberculosis in non-human primates:protection associated with mycobacterial antigen-specific CD4 effector memory T-cell populations. Tuberculosis (Edinb). 2016;101:174-190.

49. Ugolini M, et al. Recognition of microbial viability via TLR8 drives TFH cell differentiation and vaccine responses. Nat Immunol. 2018;19(4):386-396

50. Griffiths KL, et al. Targeting dendritic cells to accelerate T-cell activation overcomes a bottleneck in tuberculosis vaccine efficacy. Nat Commun. 2016;7:13894.

51. Lambrecht BN, Kool M, Willart MA, Hammad H. Mechanism of action of clinically approved adjuvants. Curr Opin Immunol. 2009;21(1):23-29.

52. Reed SG, Orr MT, Fox CB. Key roles of adjuvants in modern vaccines. Nat Med. 2013;19(12):1597-1608

53. Kool M, et al. Alum adjuvant boosts adaptive immunity by inducing uric acid and activating inflammatory dendritic cells. J Exp Med. 2008;205(4):869-882

54. O'Hagan DT, Ott GS, De Gregorio E, Seubert A. The mechanism of action of MF59 - an innately attractive adjuvant formulation. Vaccine.
2012;30(29):4341-4348.

55. Reed SG, Carter D, Casper C, Duthie MS, Fox CB. Correlates of GLA family adjuvants' activities. Semin Immunol. 2018;39:22-29.

56. Muñoz-Wolf N, Lavelle EC. A guide to IL-1 family cytokines in adjuvanticity. FEBS $J$. 2018;285(13):2377-2401.

57. Del Giudice G, Rappuoli R, Didierlaurent AM. Correlates of adjuvanticity: a review on adjuvants in licensed vaccines. Semin Immunol. 2018;39:14-21.

58. van den Berg RA, et al. Adjuvant-associated peripheral blood mRNA profiles and kinetics induced by the adjuvanted recombinant protein candidate tuberculosis vaccine M72/AS01 in Bacillus Calmette-Guérin-vaccinated adults. Front Immunol. 2018;9:564.

59. Coccia M, et al. Cellular and molecular synergy in AS01-adjuvanted vaccines results in an early IFNgamma response promoting vaccine immunogenicity. NPJ Vaccines. 2017;2:25.

60. Bertholet $S$, et al. A defined tuberculosis vaccine candidate boosts BCG and protects against multidrug-resistant Mycobacterium tuberculosis. Sci Transl Med. 2010;2(53):53ra74.

61. Coler RN, et al. The TLR-4 agonist adjuvant, GLA-SE, improves magnitude and quality of immune responses elicited by the ID93 tuberculosis vaccine: first-in-human trial. NPJVaccines. 2018;3:34.

62. Penn-Nicholson A, et al. Safety and immunogenicity of the novel tuberculosis vaccine ID93 + GLA-SE in BCG-vaccinated healthy adults in South Africa: a randomised, doubleblind, placebo-controlled phase 1 trial. Lancet Respir Med. 2018;6(4):287-298.

63. Luabeya AK, et al. First-in-human trial of the post-exposure tuberculosis vaccine H56:IC31 in Mycobacterium tuberculosis infected and non-infected healthy adults. Vaccine. 2015;33(33):4130-4140.

64. Suliman S, et al. Dose optimization of H56:IC31 vaccine for tuberculosis-endemic populations. a double-blind, placebo-controlled, dose-selection trial. Am J Respir Crit Care Med 2019;199(2):220-231.

65. Leroux-Roels I, et al. Improved CD4 ${ }^{+} \mathrm{T}$ cell responses to Mycobacterium tuberculosis in PPD-negative adults by M72/AS01 as compared to the M72/ASO2 and Mtb72F/ASO2 tuberculosis candidate vaccine formulations: a randomized trial. Vaccine. 2013;31(17):2196-2206.

66. Quintin J, et al. Candida albicans infection affords protection against reinfection via functional reprogramming of monocytes. Cell Host Microbe. 2012;12(2):223-232.

67. Cheng SC, et al. mTOR- and HIF-1 $\alpha$-mediated aerobic glycolysis as metabolic basis for trained immunity. Science. 2014;345(6204):1250684.

68. Arts RJ, et al. Glutaminolysis and fumarate accumulation integrate immunometabolic and epigenetic programs in trained immunity. Cell Metab. 2016;24(6):807-819.

69. Bekkering S, et al. Metabolic induction of trained immunity through the mevalonate pathway. Cell. 2018;172(1-2):135-146.e9.

70. Bierne H, Hamon M, Cossart P. Epigenetics and bacterial infections. Cold Spring Harb Perspect Med. 2012;2(12):a010272.

71. Monticelli S, Natoli G. Short-term memory of danger signals and environmental stimuli in immune cells. Nat Immunol. 2013;14(8):777-784.

72. Bhatt DM, et al. Transcript dynamics of proinflammatory genes revealed by sequence analysis of subcellular RNA fractions. Cell. 2012;150(2):279-290.

73. Jin F, et al. A high-resolution map of the threedimensional chromatin interactome in human cells. Nature. 2013;503(7475):290-294.

74. Saeed S, et al. Epigenetic programming of monocyte-to-macrophage differentiation and trained innate immunity. Science. 2014;345(6204):1251086.

75. Fanucchi S, et al. Immune genes are primed for robust transcription by proximal long noncoding RNAs located in nuclear compartments. Nat Genet. 2019;51(1):138-150.

76. Li G, et al. Extensive promoter-centered chromatin interactions provide a topological basis for transcription regulation. Cell. 2012;148(1-2):84-98.

77. Gomez JA, et al. The NeST long ncRNA controls microbial susceptibility and epigenetic activation of the interferon- $\gamma$ locus. Cell. 2013;152(4):743-754.

78. Wang KC, et al. A long noncoding RNA maintains active chromatin to coordinate homeotic gene expression. Nature. 2011;472(7341):120-124.

79. Smith JN, Kanwar VS, MacNamara KC. Hematopoietic stem cell regulation by Type I and II interferons in the pathogenesis of acquired aplastic anemia. Front Immunol. 2016;7:330.

80. Shahbazian LM, Quinton LJ, Bagby GJ, Nelson S, Wang G, Zhang P. Escherichia coli pneumonia enhances granulopoiesis and the mobilization of myeloid progenitor cells into the systemic circulation. Crit Care Med. 2004;32(8):1740-1746.

81. Baldridge MT, King KY, Boles NC, Weksberg DC, Goodell MA. Quiescent haematopoietic stem cells are activated by IFN-gamma in response to chronic infection. Nature. 2010;465(7299):793-797.

82. Kaufmann E, et al. BCG educates hematopoietic stem cells to generate protective innate immunity against tuberculosis. Cell. 2018;172(1-2):176-190.e19.

83. Mitroulis I, et al. Modulation of myelopoiesis progenitors is an integral component of trained immunity. Cell. 2018;172(1-2):147-161.e12

84. Essers MAG, et al. IFN $\alpha$ activates dormant haematopoietic stem cells in vivo. Nature. 2009;458(7240):904-908

85. Hartner JC, Walkley CR, Lu J, Orkin SH. ADAR1 is essential for the maintenance of hematopoiesis and suppression of interferon signaling. Nat Immunol. 2009;10(1):109-115.

86. Sato T, Onai N, Yoshihara H, Arai F, Suda T, Ohteki T. Interferon regulatory factor-2 protects quiescent hematopoietic stem cells from type I interferon-dependent exhaustion. Nat Med. 2009;15(6):696-700.

87. King KY, Goodell MA. Inflammatory modulation of HSCs: viewing the HSC as a foundation for the immune response. Nat Rev Immunol. 2011;11(10):685-692.

88. Kolb-Mäurer A, Wilhelm M, Weissinger F, Bröcker $\mathrm{E}-\mathrm{B}$, Goebel W. Interaction of human hematopoietic stem cells with bacterial pathogens. Blood. 2002;100(10):3703-3709.

89. Reece ST, et al. Mycobacterium tuberculosisinfected hematopoietic stem and progenitor cells 
unable to express inducible nitric oxide synthase propagate tuberculosis in mice. JInfect Dis. 2018;217(10):1667-1671.

90. Tornack J, et al. Human and mouse hematopoietic stem cells are a depot for dormant mycobacterium tuberculosis. PLOS ONE. 2017;12(1):e0169119.

91. Das B, et al. CD271(+) bone marrow mesenchymal stem cells may provide a niche for dormant Mycobacterium tuberculosis. Sci Transl Med. 2013;5(170):170ra13.

92. Tso GH, Law HK, Tu W, Chan GC, Lau YL. Phagocytosis of apoptotic cells modulates mesenchymal stem cells osteogenic differentiation to enhance IL-17 and RANKL expression on CD4+ T cells. Stem Cells. 2010;28(5):939-954.

93. Behr MA, Divangahi M. Freund's adjuvant, NOD2 and mycobacteria. Curr Opin Microbiol. 2015;23:126-132.

94. Kleinnijenhuis J, et al. Bacille Calmette-Guerin induces NOD2-dependent nonspecific protection from reinfection via epigenetic reprogramming of monocytes. Proc Natl Acad Sci U S A. 2012;109(43):17537-17542.

95. Nagai Y, et al. Toll-like receptors on hematopoietic progenitor cells stimulate innate immune system replenishment. Immunity. 2006;24(6):801-812.

96. Yáñez A, Goodridge HS, Gozalbo D, Gil ML. TLRs control hematopoiesis during infection. Eur JImmunol. 2013;43(10):2526-2533.

97. Belyaev NN, et al. Induction of an IL7-R+c-Kithi myelolymphoid progenitor critically dependent on IFN- $\gamma$ signaling during acute malaria. Nat Immunol. 2010;11(6):477-485.

98. Flynn JL, Chan J, Triebold KJ, Dalton DK, Stewart TA, Bloom BR. An essential role for interferon gamma in resistance to Mycobacterium tuberculosis infection. JExp Med.1993;178(6):2249-2254.

99. Mayer-Barber KD, et al. Host-directed therapy of tuberculosis based on interleukin-1 and type I interferon crosstalk. Nature. 2014;511(7507):99-103.

100.Doolan DL, Hoffman SL. IL-12 and NK cells are required for antigen-specific adaptive immunity against malaria initiated by CD8+ T cells in the Plasmodium yoelii model. J Immunol. 1999;163(2):884-892.

101.Garcia-Peñarrubia P, Koster FT, Kelley RO, McDowell TD, Bankhurst AD. Antibacterial activity of human natural killer cells. J Exp Med. 1989;169(1):99-113.

102. Orange JS, Wang B, Terhorst C, Biron CA. Requirement for natural killer cell-produced interferon gamma in defense against murine cytomegalovirus infection and enhancement of this defense pathway by interleukin 12 adminis- tration. J Exp Med. 1995;182(4):1045-1056.

103. Tay CH, Szomolanyi-Tsuda E, Welsh RM. Control of infections by NK cells. In: Kärre K, Colonna M eds. Specificity, Function, and Development of NK Cells; NK Cells: The Effector Arm of Innate Immunity Berlin, Heidelberg, Germany: SpringerVerlag; 1998:193-220.

104.Vankayalapati R, et al. Role of NK cell-activating receptors and their ligands in the lysis of mononuclear phagocytes infected with an intracellular bacterium. JImmunol. 2005;175(7):4611-4617.

105. Vankayalapati R, et al. The NKp46 receptor contributes to NK cell lysis of mononuclear phagocytes infected with an intracellular bacterium. J Immunol. 2002;168(7):3451-3457.

106.Schierloh P, et al. Increased susceptibility to apoptosis of CD56dimCD16+ NK cells induces the enrichment of IFN- $\gamma$-producing CD56bright cells in tuberculous pleurisy. JImmunol. 2005;175(10):6852-6860

107. Fu X, et al. Human natural killer cells expressing the memory-associated marker CD45RO from tuberculous pleurisy respond more strongly and rapidly than CD45RO- natural killer cells following stimulation with interleukin-12. Immunology. 2011;134(1):41-49.

108. Roy S, Barnes PF, Garg A, Wu S, Cosman D, Vankayalapati R. NK cells lyse T regulatory cells that expand in response to an intracellular pathogen. J Immunol. 2008;180(3):1729-1736.

109. Dhiman R, et al. NK1.1+ cells and IL-22 regulate vaccine-induced protective immunity against challenge with Mycobacterium tuberculosis. J Immunol. 2012;189(2):897-905.

110.Venkatasubramanian S, et al. IL-21-dependent expansion of memory-like NK cells enhances protective immune responses against Mycobacterium tuberculosis. Mucosal Immunol. 2017;10(4):1031-1042.

111. Roy Chowdhury R, et al. A multi-cohort study of the immune factors associated with M. tuberculosis infection outcomes. Nature. 2018;560(7720):644-648.

112. Kagina BM, et al. Delaying BCG vaccination from birth to 10 weeks of age may result in an enhanced memory CD4 T cell response. Vaccine. 2009;27(40):5488-5495.

113. Paust S, et al. Critical role for the chemokine receptor CXCR6 in NK cell-mediated antigenspecific memory of haptens and viruses. Nat Immunol. 2010;11(12):1127-1135.

114. Sun JC, Lanier LL. Versatility in NK cell memory. Immunol Cell Biol. 2011;89(3):327-329.

115. Sun JC, Lopez-Verges S, Kim CC, DeRisi JL, Lanier LL. NK cells and immune "memory."
Jimmunol. 2011;186(4):1891-1897.

116. Sutherland I, Lindgren I. The protective effect of BCG vaccination as indicated by autopsy studies. Tubercle. 1979;60(4):225-231.

117. Soysal A, et al. Effect of BCG vaccination on risk of Mycobacterium tuberculosis infection in children with household tuberculosis contact: a prospective community-based study. Lancet. 2005;366(9495):1443-1451.

118. Roy A, et al. Effect of BCG vaccination against Mycobacterium tuberculosis infection in children: systematic review and meta-analysis. BMJ. 2014;349:g4643.

119. Hill PC, et al. Longitudinal assessment of an ELISPOT test for Mycobacterium tuberculosis infection. PLoS Med. 2007;4(6):e192.

120. Verrall AJ, et al. Early clearance of Mycobacterium tuberculosis is associated with increased innate immune responses [published online ahead of print April 8, 2019]. J Infect Dis. https:// doi.org/10.1093/infdis/jiz147.

121. [No authors listed]. BCG: bad news from India. Lancet. 1980;315(8159):73-74.

122. Mangtani $P$, et al. The duration of protection of school-aged BCG vaccination in England: a population-based case-control study. Int J Epidemiol. 2018;47(1):193-201.

123. Nguipdop-Djomo P, Heldal E, Rodrigues LC, Abubakar I, Mangtani P. Duration of BCG protection against tuberculosis and change in effectiveness with time since vaccination in Norway: a retrospective population-based cohort study. Lancet Infect Dis. 2016;16(2):219-226.

124.de Bree C, van Crevel R, Netea MG. H4:IC31 vaccine or BCG revaccination for tuberculosis. NEngl J Med. 2018;379(20):1969.

125. Benn CS, Netea MG, Selin LK, Aaby P. A small jab - a big effect: nonspecific immunomodulation by vaccines. Trends Immunol. 2013;34(9):431-439.

126. Kleinnijenhuis J, et al. Long-lasting effects of BCG vaccination on both heterologous Th1/Th17 responses and innate trained immunity. JInnate Immun. 2014;6(2):152-158.

127. Arts RJW, et al. BCG vaccination protects against experimental viral infection in humans through the induction of cytokines associated with trained immunity. Cell Host Microbe. 2018;23(1):89-100.e5.

128. Kleinnijenhuis J, van Crevel R, Netea MG. Trained immunity: consequences for the heterologous effects of BCG vaccination. Trans $R$ Soc Trop Med Hyg. 2015;109(1):29-35.

129. Burberry A, et al. Infection mobilizes hematopoietic stem cells through cooperative NOD-like receptor and Toll-like receptor signaling. Cell Host Microbe. 2014;15(6):779-791. 